\title{
Seizure in Pregnancy Following Cerebral Venous Sinus Thrombosis
}

\author{
Farnoush Farzi $^{1,} ;$ Mehrsima Abdollahzadeh ${ }^{1}$; Roya Faraji $^{2}$; Tahereh Chavoushi $^{1}$ \\ ${ }^{1}$ Department of Anesthesiology, Reproductive Health Research Center, Guilan University of Medical Sciences, Rasht, Iran \\ ${ }^{2}$ Department of Obstetrics and Gynecology, Reproductive Health Research Center, Guilan University of Medical Sciences, Rasht, Iran \\ ${ }^{*}$ Corresponding author: Farnoush Farzi, Department of Anesthesiology, Reproductive Health Research Center, Guilan University of Medical Sciences, Rasht, Iran. Tel: +98-9113311653, \\ Fax:+98-1333325624, E-mail: Farnoush_farzi@yahoo.com
}

Received: January 14, 2015; Revised: March 7, 2015; Accepted: April 22, 2015

\begin{abstract}
Introduction: Seizure involves less than $1 \%$ of pregnancies; however it is associated with increased maternal and fetal complications. Cerebral venous sinus thrombosis is a rare, but potentially life-threatening cause of seizure during pregnancy, presenting primarily as seizure in $12 \%-31.9 \%$ of cases. Pregnancy and puerperium are known as the risk factors of cerebral venous sinus thrombosis.

Case Presentation: Here is presented a case of seizure after delivery by cesarean section in an otherwise healthy woman. The final diagnosis was cerebral venous sinus thrombosis probably due to hypercoagulable state in pregnancy.

Conclusions: If seizure occurs during the peripartum period, along with providing complete cardiovascular and respiratory support, advanced diagnostic measures are needed and cerebral venous sinus thrombosis should be considered as a possible diagnosis.
\end{abstract}

Keywords: Seizure; Pregnancy; Thrombosis

\section{Introduction}

Seizure involves less than $1 \%$ of pregnancies; however, it is associated with increased maternal and fetal complications. When seizure occurs for the first time during pregnancy, investigations should include obtaining careful history, physical examination, and appropriate tests. Moreover, all potential causes of seizure in pregnancy should be taken into consideration including: epilepsy, cerebrovascular events (hemorrhage, ruptured aneurysm, arterial embolism or thrombosis, cerebral venous sinus thrombosis, ischemic hypoxic encephalopathy, angioma), eclampsia, congenital cerebral defects, encephalitis, trauma, brain tumors, hepatic and renal failure, metabolic abnormalities (hypoglycemia, hyponatremia, hyperosmolar conditions, and hypokalemia), drug overdose/withdrawal, thrombophilia, antiphospholipid syndrome, and autoimmune abnormalities (systemic lupus erythematosus, thrombotic thrombocytopenic purpura) (1).

Cerebral Venous Sinus Thrombosis (CVST) is a rare complication with estimated occurrence of 3 - 5/1000000 in general population $(2,3)$, that rarely induces cerebral hemorrhage (4). It is also a rare but potentially life-threatening cause of seizure during pregnancy, presenting primarily as seizure in $12 \%-31.9 \%$ of cases. In other cases, headache, focal and generalized convulsions, unilateral and bilateral paresis, and papilledema can occur (3). Pregnancy and puerperium are known as CVST risk factors (5). One significant reason is the hypercoagulable state due to increased levels of coagulation factors in pregnancy.
The activity of the majority of coagulation factors (I, VII, VIII, IX, X, XII) increase during pregnancy, whereas the activity of physiologic anticoagulants decrease (6).

\section{Case Presentation}

A 21-year-old healthy primigravida woman referred for emergent cesarean section due to Intrauterine Growth Retardation (IUGR) and fetal distress. Routine monitoring including pulse oximetry, noninvasive blood pressure, and ECG was established and cesarean section was performed under spinal anesthesia by hyperbaric 5\% lidocaine $70 \mathrm{mg}$ in sitting position from L3-L4 inter vertebral space by 25 gauge Quincke needle. Before and during surgery and in the recovery room, the patient was hemodynamically stable with blood pressure in range of 115/65 before surgery and 90/55 to 115/78 during and after surgery. She received $500 \mathrm{~mL}$ ringer lactate solution before neuraxial block and $2 \mathrm{~L}$ during surgery, estimated blood loss was $500 \mathrm{~mL}$. Oxytocin 40 IU as continuous infusion was administered in the first hour after delivery. She also had normal conscious level. Three hours after transmission to the ward, the patient had generalized tonic-colonic seizure lasting less than one minute. The patient was immediately transmitted to ICU (intensive care unit) and had another generalized tonic-colonic seizure just after transmission that lasted for more than one minute and was controlled by administering $10 \mathrm{mg}$ diazepam IV (intravenous), then the patient entered into the postictal phase. Five minutes later, seizure occurred

Copyright (C) 2015, Iranian Society of Regional Anesthesia and Pain Medicine(ISRAPM). This is an open-access article distributed under the terms of the Creative Commons Attribution-NonCommercial 4.0 International License (http://creativecommons.org/licenses/by-nc/4.0/) which permits copy and redistribute the material just in noncommercial usages, provided the original work is properly cited. 
Farzi F et al.

again and the patient was intubated and infusion of sodium thiopental $1.5 \mathrm{mg} / \mathrm{kg} / \mathrm{h}$ started due to resistant seizures. Meanwhile, the consultant neurologist administered $750 \mathrm{mg}$ phenytoin and $800 \mathrm{mg}$ Depakine, and as eclampsia was suspected, the obstetrician administered magnesium sulfate $4 \mathrm{~g}$ bolus intravenously in 10 minutes followed by infusion of $2 \mathrm{~g} / \mathrm{h}$. In the ICU blood and urine samples were taken. Blood glucose level was normal and ABG (arterial blood gas) analysis showed metabolic acidosis that was corrected by administering sodium bicarbonate. Hypokalemia $(\mathrm{K}=3.1)$ and hypocalcemia $(\mathrm{Ca}=7.1)$ were observed in primary laboratory tests that were corrected with intravenous infusion of potassium $10 \mathrm{mEq} / \mathrm{h}$ and slow IV injection of calcium $1 \mathrm{~g}$. Cell blood count, PTT (partial thromboplastin time), PT (prothrombin time), INR (international normalized ratio) and liver function tests were in normal range $(\mathrm{AST}=35 \mathrm{U} / \mathrm{L}, \mathrm{ALT}=42 \mathrm{U} / \mathrm{L}, \mathrm{PT}$ $=13$ seconds, $\mathrm{PTT}=30$ seconds, $\mathrm{INR}=1.2$, proteinuria $=$ negative). On blood pressure monitoring, she always had normal systolic, diastolic and mean levels. Therefore, eclampsia and HELLP (Hemolysis, Elevated Liver enzymes, Low Platelet count) syndrome were excluded and other causes of seizure were thought more probable.

On ophthalmic examination, there was no sign of papilledema. The patient had not hemiparesis, hemiplegia or cranial nerve involvement on neurologic examination.

Despite all treatments, the patient had three more seizures in 1-1.5 hour(s) intervals that were controlled by injection of $100 \mathrm{mg}$ sodium thiopental. Six hours after the last seizure, infusion of sodium thiopental was reduced to $1 \mathrm{mg} / \mathrm{kg} / \mathrm{h}$ and after 36 hours infusion of sodium thiopental was stopped and the patient was extubated. After extubation, the patient was a little lethargic; however, she gained complete consciousness within 6 - 8 hours later.

As the seizures were not controlled by magnesium sulfate, neurologic problems were considered and in the second day brain MRI (magnetic resonance imaging) was performed that revealed obstruction of the cerebral venous plexus (Figure 1). Immediately, anticoagulation therapy was established by Celexane $40 \mathrm{mg}$ twice daily. Four days later, magnetic resonance venogram (MRV) showed that the obstruction was resolved.

After confirming the diagnosis of cerebral venous sinus thrombosis, some laboratory screening tests including antinuclear antibody (ANA), anti-neutrophil cytoplasmic antibodies (perinuclear and cytoplasmic) [ANCA(P.C)], anti-double strain DNA (anti DSDNA), Protein C and S, C3, C4, CH50 (50\% hemolytic complement), anti-thrombin 3 and rheumatoid factor (RF) were performed to evaluate coagulation state; all showed normal values. Based on the normal laboratory results and no previous history of coagulopathies in the patient and her relatives, the final diagnosis was cerebral venous sinus thrombosis probably due to hypercoagulable state in pregnancy.

After 11 days, the patient was discharged in good health condition without any neurological complications with warfarin prescription.

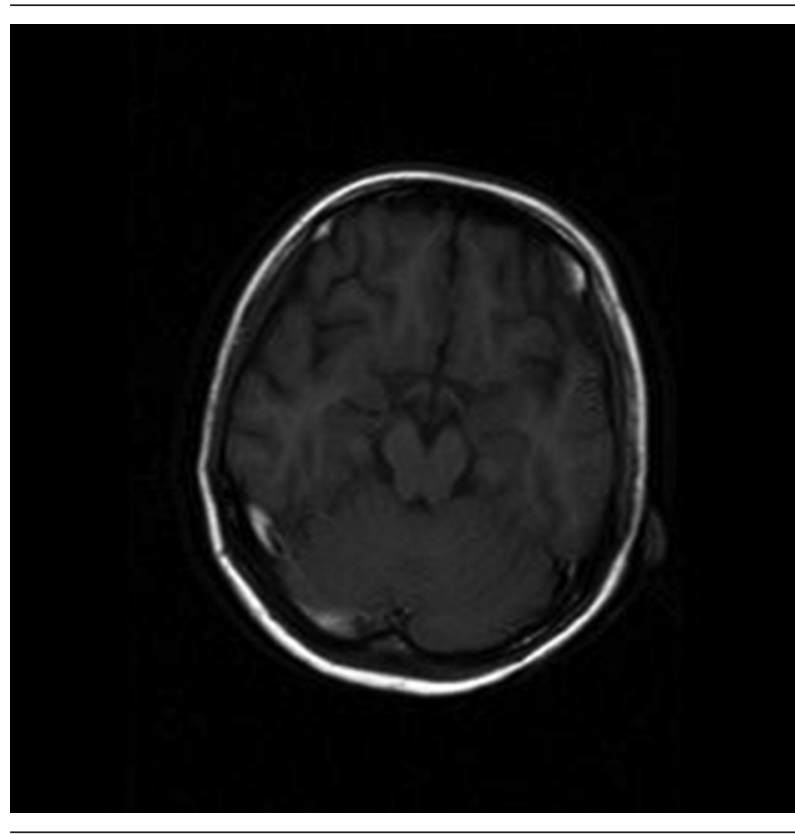

Figure 1. Remarked Thrombosis in Right Transverse Sinus (T1, in the sec ond day of seizure and loss of consciousness)

\section{Discussion}

Seizure in pregnancy is associated with maternal and fetal complications. The differential diagnosis of seizure in pregnant women consists of eclampsia, epilepsy, cerebral vascular accident, and CVST.

In this subject, magnesium sulfate therapy was initiated due to the primary diagnosis of eclampsia; however despite administering the loading and maintenance doses, seizures did not stop. Therefore, further investigations including MRI and MRV revealed CVST. Anticoagulation therapy resulted in complete recovery of the patient.

Venous thrombosis often occurs in lower limbs and can result in pulmonary artery embolism (7). Sometimes, thrombosis occurs in rare locations such as cerebral venous sinus. Hereditary thrombophilia (such as mutation of factor V Leiden or hereditary protein $\mathrm{C}$ and protein $\mathrm{S}$ deficiency), acquired hyper thrombotic conditions, systemic diseases (vasculitis such as behcet syndrome or systemic lupus erythematosus), neoplasms, systemic infectious diseases, local reasons such as mastoiditis and otitis, and oral contraceptives are CVST developing risk factors (8).

Sidhom et al. conducted a study to investigate presentations and risk factors of cerebral venous sinus thrombosis. In $29 \%$ of the patients, seizure was the first sign, of which $59 \%$ had a generalized seizure. Risk factors included pregnancy $9 \%$, puerperium period $29 \%$ and coagulopathies 59\% in their cases (9); as mentioned, in the current subject the risk factor of CVST was pregnancy.

Sharpe et al. reported a woman with the history of CVST in her first degree relative. According to the screening test she had hereditary antithrombin I deficiency. As the patient decided to become pregnant, prophylaxis 
with Low Molecular Weight Heparin (LMVH) initiated and there was no problem until the 34th week of gestation that the patient complained about headache and vomiting that lasted for four days. In the physical examination there was no neurologic finding and the fetus was not in the stress condition in sonography. Brain CT (computed tomography) scan showed a hypo density in the left transverse sinus, suggesting thrombosis; brain MRI was normal; MRV revealed filling defects in the sigmoid transverse sinus and a part of the base of skull from left internal jugular vein, suggesting dural venous sinus thrombosis with no evidence of infarction. Treatment with LMVH was stopped and unfractionated heparin was administered to achieve a PTT of $60-80$ s. Eight hours before cesarean section, heparin was withheld, resuming 12 hours after surgery. One day after cesarean section, warfarin was administrated to achieve INR $=2-3$. Then, two days later, heparin was discontinued. Furthermore, concentrated plasma antithrombin was continued until six weeks after delivery (10). In the current subject, coagulation studies were conducted and all were in normal range.

Soydinc et al. presented a case of CVST on the basis of preeclampsia with headache, neurologic symptoms and signs and ultimately seizure after delivery which ended up with maternal mortality due to misdiagnosing as eclampsia. They claimed that diagnosis of this entity was difficult because of variable nature of its occurrence (3).

Dag et al. reported a patient with factor V Leiden mutation with CVST at 24th week of pregnancy that was diagnosed as HELLP syndrome at 34th week of pregnancy (11).

Nie et al. reported a case of CVST with hemorrhage in early pregnancy that was treated with anticoagulant and favorable results were obtained (4).

Yamamoto et al. investigated a subject with CVST in the ninth week of pregnancy with generalized seizure and severe SAH (subarachnoid hemorrhage) that needed decompression craniotomy and intrauterine curettage and had an excellent recovery after two months with only a slight visual field defect. They wanted to emphasize on misdiagnosing of CVST in the early pregnancy (12).

The best conclusion that can be drawn is that, if seizure occurs during the peripartum period, as definite diagnosis is not clear at the early presentation, along with providing complete supportive neurologic, cardiovascular and respiratory cares, advanced diagnostic measures should be performed to diagnose and manage the predisposing factors. Cerebral venous sinus thrombosis should be considered as a rare but possible hazardous cause that deteriorates maternal and fetal outcomes.

\section{Acknowledgements}

The authors would like to thank Doctor Aliya Saberi, neurologist, and Ms. Seyede Fateme Heirati for their cooperation.

\section{Authors' Contributions}

Manuscript preparation: Farnoush Farzi, Mehrsima Abdollahzadeh, Roya Faraji and Tahereh Chavoushi.

\section{References}

1. Hart LA, Sibai BM. Seizures in pregnancy: epilepsy, eclampsia, and stroke. Semin Perinatol. 2013;37(4):207-24.

2. McBane R2, Tafur A, Wysokinski WE. Acquired and congenital risk factors associated with cerebral venous sinus thrombosis. Thromb Res. 2010;126(2):81-7.

3. Soydinc HE, Ozler A, Evsen MS, Sak ME, Turgut A, Basaranoglu S, et al. A case of cerebral sinus venous thrombosis resulting in mortality in severe preeclamptic pregnant woman. Case Rep Obstet Gynecol. 2013;2013:402601.

4. Nie Q, Guo P, Ge J, Qiu Y. Cerebral venous sinus thrombosis with cerebral hemorrhage during early pregnancy. Neurosciences (Riyadh). 2015;20(1):48-51.

5. Kalita J, Chandra S, Misra UK. Significance of seizure in cerebral venous sinus thrombosis. Seizure. 2012;21(8):639-42.

6. Hines R.L M. K. New York: Elsevier; 2012. Anesthesia and Co-exiting DiseaseHines R.L M.

7. Heit JA. The epidemiology of venous thromboembolism in the community. Arterioscler Thromb Vasc Biol. 2008;28(3):370-2.

8. Demir CF, Inci MF, Ozkan F, Yildiz M, Ozdemir H. Clinical and radiological management and outcome of pregnancies complicated by cerebral venous thrombosis: a review of 19 cases.J Stroke Cerebrovasc Dis. 2013;22(8):1252-7.

9. Sidhom Y, Mansour M, Messelmani M, Derbali H, Fekih-Mrissa $\mathrm{N}$, Zaouali J, et al. Cerebral venous thrombosis: clinical features, risk factors, and long-term outcome in a Tunisian cohort. J Stroke Cerebrovasc Dis. 2014;23(6):1291-5.

10. Sharpe CJ, Crowther MA, Webert KE, Donnery C. Cerebral venous thrombosis during pregnancy in the setting of type I antithrombin deficiency: case report and literature review. Transfus Med Rev. 2011;25(1):61-5.

11. Dag ZO, Isik Y, Simsek Y, Tulmac OB, Demiray D. HELLP Syndrome and Cerebral Venous Sinus Thrombosis Associated with Factor V Leiden Mutation during Pregnancy. Case Rep Obstet Gynecol. 2014;2014:582890.

12. Yamamoto J, Kakeda S, Takahashi M, Idei M, Nakano Y, Soejima Y, et al. Severe subarachnoid hemorrhage associated with cerebral venous thrombosis in early pregnancy: a case report. J Emerg Med.2013;45(6):849-55. 